\title{
Aspecto psicossocial do ambiente de trabalho de profissionais de enfermagem segundo o modelo demanda-controle
}

\author{
Psychosocial aspect of nursing personnel's work environment according to the demand-control model \\ Aspecto psicosocial del ambiente de trabajo del personal de enfermería según el modelo demanda control
}

\author{
Lucas Carvalho Santana'@; Lúcia Aparecia Ferreira' ${ }^{\circledR}$; Marli Aparecida Reis Coimbra' $\odot$; \\ Marina Pereira Rezende' ${ }^{\circledR}$; Cíntia Machado Dutra'`๑
}

'Universidade Federal do Triângulo Mineiro, Uberaba, MG, Brasil

\begin{abstract}
RESUMO
Objetivo: avaliar o aspecto psicossocial do ambiente de trabalho de profissionais de enfermagem de um hospital universitário. Método: estudo transversal, exploratório e quantitativo realizado com 124 profissionais de enfermagem de um hospital universitário do interior de Minas Gerais, A coleta de dados foi realizada entre os meses de julho e setembro de 2018 e utilizouse a Job Stress Scale (JSS). O presente estudo foi aprovado pelo CEP/UFTM. Resultados: os profissionais foram alocados nos quadrantes do modelo Demanda-Controle e identificou-se a seguinte prevalência: $30,6 \%$ na situação de alta exigência; $28,2 \%$ na de baixa exigência; $21,8 \%$ de trabalho ativo e $19,4 \%$ na situação de trabalho passivo. Conclusão: a avaliação do aspecto psicossocial do ambiente de trabalho evidenciou maiores taxas de indivíduos em situação de trabalho de alta exigência entre profissionais de enfermagem atuantes nas enfermarias $(41,9 \%)$ e no setor de bloco cirúrgico $(33,3 \%)$.

Descritores: Profissionais de Enfermagem; Ambiente de Trabalho; Estresse Ocupacional; Saúde do trabalhador
\end{abstract}

\section{ABSTRACT}

Objective: to evaluate the psychosocial aspect of the work environment of nursing personnel at a university hospital. Method: in this exploratory, quantitative, cross-sectional study of 124 nursing professionals at a university hospital in Minas Gerais state, data were collected between July and September 2018. The Job Stress Scale (JSS) was used. The study was approved by the research ethics committee of the Universidade Federal do Triângulo Mineiro. Results: participants were allocated to quadrants of the Demand-Control model, and the following prevalences were identified: $30.6 \%$ in high-stress situations; $28.2 \%$ in lowstress; $21.8 \%$ in active work; and $19.4 \%$ in passive work. Conclusion: assessment of the psychosocial aspect of the work environment found higher rates of individuals in high-stress jobs among nursing personnel on the wards (41.9\%) and in the surgical sector (33.3\%).

Descriptors: Nurse Practitioners; Working Environment; Occupational Stress; Occupational health.

\section{RESUMEN}

Objetivo: evaluar el aspecto psicosocial del entorno laboral del personal de enfermería de un hospital universitario. Método: en este estudio exploratorio, cuantitativo y transversal de 124 profesionales de enfermería de un hospital universitario del estado de Minas Gerais, se recolectaron datos entre julio y septiembre de 2018. Se utilizó la Job Stress Scale (JSS). El estudio fue aprobado por el comité de ética en investigación de la Universidade Federal do Triângulo Mineiro. Resultados: los participantes fueron asignados a cuadrantes del modelo Demanda-Control, y se identificaron las siguientes prevalencias: 30,6\% en situaciones de alto estrés; $28,2 \%$ en bajo estrés; $21,8 \%$ en trabajo activo; y el $19,4 \%$ en trabajo pasivo. Conclusión: la evaluación del aspecto psicosocial del ambiente de trabajo encontró tasas más altas de individuos en trabajos de alto estrés entre el personal de enfermería en las salas $(41,9 \%)$ y en el sector quirúrgico $(33,3 \%)$.

Descriptores: Enfermeras Practicantes; Ambiente de Trabajo; Estrés Laboral; Salud laboral.

\section{INTRODUÇÃO}

No âmbito da saúde do trabalhador, estudos têm destacado a inexistência de relações de neutralidade entre o trabalho e o processo saúde/doença, a qual reforça a concepção de que toda atividade produtiva possui potencial para promover saúde ou produzir doença, dependendo de como se configuram os elementos da organização, do processo de trabalho e o modo como se articulam com características subjetivas do trabalhador ${ }^{1,2}$.

O ambiente de trabalho possui aspectos ocupacionais que ocasionam riscos, integram o processo de trabalho e se relacionam com os elementos laborais, o que pode produzir sobrecarga psíquica nos indivíduos e são capazes de gerar sofrimento e adoecimento. Dentre esses aspectos, pode-se citar os psicossociais, pois são complexos e envolvem características relacionadas ao indivíduo (personalidade), ao ambiente de trabalho (demandas e controle sobre a tarefa) e ao ambiente social (fatores culturais) ${ }^{3,4}$.

Autor correspondente: Lucas Carvalho Santana·E-mail: lucas_enfer@hotmail.com.

Editora responsável: Helena Maria Scherlowski Leal David 
Alguns autores evidenciaram a importância do Modelo Demanda-Controle na investigação do ambiente psicossocial do trabalho e os efeitos do estresse ocupacional sobre a saúde, abordando suas diversas repercussões. $A$ principal predição desse modelo é que o trabalho realizado na situação de alta exigência representa risco à saúde, favorecendo o adoecimento físico e mental dos trabalhadores ${ }^{4-7}$.

O modelo em questão é aceito internacionalmente pela comunidade acadêmica e amplamente utilizada em pesquisas científicas relacionadas ao tema ${ }^{6,8-11}$.

Os fatores psicossociais têm sido relacionados ao surgimento de doenças cardiovasculares, distúrbios osteomusculares, alteração na saúde mental, e, possivelmente, acarreta na diminuição da capacidade para o trabalho ${ }^{4,12,13}$. Ademais, gera repercussões na vida do trabalhador, afetando diretamente a saúde e a qualidade de vida, tendo como consequências absenteísmo, baixo desempenho, alta rotatividade, e violência no local de trabalho 1,14,15.

A realização de estudos na área da saúde ocupacional de profissionais de enfermagem, sobretudo da análise do ambiente psicossocial do trabalho é importante pela natureza das atividades que estes profissionais executam, uma vez que a qualidade e eficácia do seu trabalho podem ter um impacto decisivo na saúde dos pacientes. Além disso, os profissionais de enfermagem estão continuamente expostos a situações de pressão, sobrecarga de trabalho e condições de trabalho precárias.

Diante do contexto, o objetivo do artigo é avaliar o aspecto psicossocial do ambiente de trabalho de profissionais de enfermagem de um hospital universitário.

\section{REFERENCIAL TEÓRICO}

O modelo Demanda-Controle foi proposto por Robert Karasek no final da década de 1970 e relaciona dois aspectos psicossociais no ambiente de trabalho ao risco de adoecimento: demanda psicológica e controle do trabalhador sobre o labor ${ }^{6,16}$.

A demanda psicológica é aquela relacionada às exigências psicológicas que o trabalhador enfrenta para concretizar suas atividades laborais. O controle do trabalhador sobre o trabalho relaciona-se a dois aspectos: ao uso de habilidades (o grau que o trabalho envolve a aprendizagem, repetitividade e criatividade) e a autonomia no processo de trabalho (habilidade para a tomada de decisões no trabalho e influências na política gerencial) ${ }^{17,18}$.

Avalia-se o ambiente psicossocial do trabalho a partir da combinação de níveis altos e baixos das dimensões demanda e controle, estabelecendo quatro situações específicas de trabalho que sugerem riscos diferenciados à saúde do trabalhador: alta exigência, trabalho ativo, trabalho passivo e baixa exigência ${ }^{6,7}$.

A situação de alta exigência (alta demanda e baixo controle) é a que apresenta reações adversas de maior desgaste psicológico; o trabalho ativo (alta demanda psicológica e alto controle) permite ao profissional ter uma ampla possibilidade de decisão sobre como e quando desenvolver suas tarefas, bem como usar sua potencialidade intelectual com esta finalidade; o trabalho passivo (baixa demanda psicológica e baixo controle) produz uma atrofia gradual de aprendizagem de habilidades; e a situação de baixa exigência (combina baixa demanda e alto controle) configura-se num estado altamente confortável e ideal de trabalho ${ }^{7}$.

Posteriormente, a inserção de uma terceira dimensão foi proposta por Johnson, ao considerar que o apoio social atuaria como importante moderador de impacto da demanda, configurando um relevante sistema de proteção dos trabalhadores contra as pressões no ambiente de trabalho. Assim, o apoio social no trabalho foi incorporado como terceiro elemento para análise ao modelo demanda- controle ${ }^{4}$.

Apoio social no trabalho refere-se à interação social existente no ambiente de trabalho, entre colegas e chefias, para cooperação na realização das atividades laborais. Possui importante papel na diminuição do desgaste sobre o trabalhador e dos riscos à saúde ${ }^{16,19}$

De acordo com os pressupostos desse modelo, o estresse ocupacional é resultante da interação entre altas demandas psicológicas e menor controle no processo de produção do trabalho, além de menor apoio social recebido de colegas e chefes. Essa condição pode acarretar consequências nocivas à saúde física ou mental do trabalhador ${ }^{18}$.

Pode-se dizer ainda que o desequilíbrio entre a demanda psicológica e o controle sobre o processo produtivo resulta em desgaste, perda de habilidades e do interesse, afetando a saúde do trabalhador ${ }^{20}$.

\section{MÉTOdo}

Trata-se de um estudo transversal, de natureza descritiva e exploratória com enfoque quantitativo realizado num hospital universitário do interior de Minas Gerais entre os meses de julho e setembro de 2018. As etapas metodológicas do estudo foram norteadas pela ferramenta STROBE. 
A população do estudo é composta por 746 profissionais da equipe de enfermagem (enfermeiros, técnicos de enfermagem e auxiliares de enfermagem) dos diversos setores da instituição. Utilizou-se como critérios de inclusão: profissionais atuantes no referido hospital e que não estavam afastados de suas atividades laborais no período de coleta de dados.

O cálculo do tamanho amostral considerou uma prevalência de exposição ao estresse ocupacional de 56,5\%, conforme estudo realizado em um hospital universitário da região sudeste do Brasil ${ }^{21}$. Ao considerar a prevalência de $56,5 \%$, uma precisão de $8 \%$ e um intervalo de confiança de $95 \%$, para uma população finita de 746 profissionais, obtevese uma amostra de 124 profissionais.

A seleção da amostra ocorreu de forma aleatória por meio do software IBM ${ }^{\circledR}$ SPSS $^{\circledR}$, versão 21 , utilizando uma lista nominal da equipe de enfermagem disponibilizada pela direção de enfermagem da instituição. Os profissionais foram convidados a participar do estudo e os que aceitaram, receberam os instrumentos de coleta de dados no próprio setor e horário de trabalho, para que eles respondessem em momento oportuno. Os instrumentos preenchidos foram recolhidos pelos pesquisadores dois dias após a entrega.

Para efetivação da coleta de dados foi utilizado um instrumento para caracterização sociodemográfica e profissional e a versão adaptada para o português da Job Stress Scale (JSS). Trata-se de uma escala elaborada originalmente na Suécia (Job Content Questionnaire) que após adaptação cultural para o português e validação foi denominada de Job Stress Scale, versão resumida. Utilizou-se este questionário por se tratar de uma escala reconhecida internacionalmente para avaliação do estresse ocupacional e dos aspectos psicossociais do trabalho baseada no modelo teórico Demanda-Controle, além de ter sido adaptada e validada para língua portuguesa ${ }^{6,16}$. Cabe mencionar que foi concedida a autorização dos autores da escala para sua utilização no estudo.

A JSS é uma escala do tipo Likert composta por 17 itens que são distribuídos em três dimensões da seguinte forma: cinco itens avaliam Demanda, seis avaliam Controle e seis a dimensão Apoio Social. Cada item da escala possui quatro alternativas de respostas com pontuação variando de um a quatro. Assim, a dimensão Demanda possui escore máximo de 2 e as dimensões Controle e Apoio Social possuem escore máximo de 24. Para cada dimensão da escala, quanto maior o escore maior a demanda, controle ou apoio social percebidos ${ }^{16}$.

Para identificação dos grupos de alta e baixa demanda e do grupo de alto e baixo controle, foi seguida a recomendação dos pesquisadores que validaram a JSS, e utilizadas as medianas das referidas dimensões como ponto de corte. Assim, os profissionais que apresentaram escores iguais ou maiores que a mediana foram enquadrados no grupo de alta demanda, controle ou apoio social; e os com escores inferiores à mediana foram alocados no grupo de baixa demanda, controle ou apoio social.

Os dados foram processados no software IBM $^{\circledR}$ SPSS $^{\circledR}$, versão 21 . Foram calculadas estatísticas descritivas por meio da utilização de medidas resumo de posição (média e mediana) e de variabilidade (amplitudes). Utilizou-se também, a análise bivariada através das medidas de associação com tabelas de contingência (risco relativo, razão de chances e respectivos intervalos de confiança), além da aplicação do teste qui-quadrado de Pearson para grupos independentes para preditores demográficos e ocupacionais dicotômicos. Este trabalho considerou um nível de significância de $5 \%(p<0,05)$.

A pesquisa seguiu as normas estabelecidas pela legislação vigente que rege sobre a realização de pesquisas com seres humanos, sendo aprovado pelo Comitê de Ética em Pesquisa da Universidade Federal do Triângulo Mineiro (CEP/UFTM) através do parecer $\mathrm{n}$ - 2.635.368. Os participantes do estudo consentiram participar do estudo mediante assinatura do TCLE, sendo orientados previamente pelo pesquisador quanto aos objetivos da pesquisa e esclarecidos que não seriam submetidos a riscos ou prejuízos, e que poderiam desistir de participar do estudo a qualquer momento.

\section{RESULTADOS E DISCUSSÃO}

A amostra foi constituída por 124 profissionais de enfermagem, conforme caracterização apresentada na Tabela 1.

Observa-se a presença majoritária de profissionais do sexo feminino (87,9\%). Dado corroborado por diversos estudos realizados com esta população de profissionais, evidenciando que a equipe de enfermagem brasileira é, estrutural e historicamente, feminina ${ }^{22-25}$.

Em relação aos dados sociodemográficos, cabe salientar também que a equipe de enfermagem estudada é composta por indivíduos jovens (76,6\% possuem até 45 anos), fato ratificado na pesquisa Perfil da Enfermagem Brasileira, onde se encontrou 74,9\% dos profissionais de enfermagem na faixa etária de até 45 anos $^{25}$.

Analisou-se a amostra conforme as três dimensões propostas pela escala utilizada: demanda, controle e apoio social. Para a realização da análise estatística bivariada, utilizou-se a mediana do escore total de cada dimensão como ponto de corte para dicotomização dessas. 
TABELA 1: Caracterização sociodemográfica e profissional da amostra ( $\mathrm{n}=124)$. Uberaba, Minas Gerais, Brasil, 2018

\begin{tabular}{|c|c|c|}
\hline Variáveis & $\mathbf{n}$ & $\%$ \\
\hline \multicolumn{3}{|l|}{ Sexo } \\
\hline Masculino & 15 & 12,1 \\
\hline Feminino & 109 & 87,9 \\
\hline \multicolumn{3}{|l|}{ Idade } \\
\hline Até 30 anos & 14 & 11,3 \\
\hline 31 a 45 anos & 81 & 65,3 \\
\hline 46 a 60 anos & 25 & 20,2 \\
\hline Acima de 60 anos & 4 & 3,2 \\
\hline \multicolumn{3}{|l|}{ Situação conjugal } \\
\hline Solteiro (a) & 38 & 30,6 \\
\hline Casado (a) ou em união estável & 66 & 53,2 \\
\hline Viúvo (a) & 1 & 0,8 \\
\hline Divorciado (a) & 19 & 15,3 \\
\hline \multicolumn{3}{|l|}{ Possui filhos? } \\
\hline Sim & 90 & 72,6 \\
\hline Não & 34 & 26,8 \\
\hline \multicolumn{3}{|l|}{ Cargo exercido } \\
\hline Auxiliares de enfermagem & 15 & 12,1 \\
\hline Técnicos de enfermagem & 85 & 68,5 \\
\hline Enfermeiros & 24 & 19,4 \\
\hline \multicolumn{3}{|l|}{ Nível de escolaridade } \\
\hline Ensino médio & 46 & 37,1 \\
\hline Superior & 38 & 30,6 \\
\hline Pós-graduação & 40 & 32,3 \\
\hline \multicolumn{3}{|l|}{ Tempo de formação } \\
\hline Até 5 anos & 6 & 4,8 \\
\hline 6 a 10 anos & 41 & 33,1 \\
\hline 11 a 15 anos & 30 & 24,2 \\
\hline 16 a 20 anos & 25 & 20,2 \\
\hline Mais de 21 anos & 22 & 17,7 \\
\hline \multicolumn{3}{|l|}{ Carga horária semanal } \\
\hline 36 horas & 90 & 72,6 \\
\hline 40 horas & 34 & 27,4 \\
\hline \multicolumn{3}{|l|}{ Horário de trabalho } \\
\hline Diurno & 63 & 50,8 \\
\hline Noturno & 61 & 49,2 \\
\hline \multicolumn{3}{|l|}{ Tempo de trabalho na instituição } \\
\hline Até 5 anos & 63 & 51,2 \\
\hline 6 a 10 anos & 16 & 13,0 \\
\hline 11 a 15 anos & 16 & 13,0 \\
\hline 16 a 20 anos & 13 & 10,6 \\
\hline Mais de 21 anos & 15 & 12,2 \\
\hline \multicolumn{3}{|l|}{ Remuneração recebida* } \\
\hline Até 2 salários & 10 & 8,2 \\
\hline 2 a 5 salários & 79 & 64,7 \\
\hline 5 a 10 salários & 31 & 25,4 \\
\hline Mais de 10 salários & 2 & 1,6 \\
\hline \multicolumn{3}{|l|}{ Presença de outro vínculo } \\
\hline Sim & 15 & 12,2 \\
\hline Não & 108 & 87,8 \\
\hline
\end{tabular}

Fonte: Dados da pesquisa, 2018

*Salário mínimo vigente no período da coleta de dados.

Nesta análise, a mediana encontrada para a dimensão demanda foi 16, para controle 16,5 e para apoio social 18 . Na literatura, são encontrados valores bem variados, haja vista que o ponto de corte para as dimensões depende de características intrínsecas à amostra estudada. Em estudo semelhante, realizado em dois hospitais públicos da região norte do Brasil, foram encontrados dados de mediana 14 para a dimensão demanda, 17 para controle e 19 para apoio 
social $^{12}$. Pesquisa realizada no estado do Rio de Janeiro identificou mediana 10 para a dimensão demanda, 12 para controle e 11 para apoio social ${ }^{26}$.

É importante mencionar que, ao comparar com os estudos citados, os profissionais de enfermagem da instituição do presente estudo apresentaram uma menor relação controle/demanda, o que implica em menos autonomia para tomada de decisão e maior desgaste psicológico. Tal situação pode contribuir para o comprometimento do rendimento do trabalhador, com baixa autonomia e poucas oportunidades de aprendizado, podendo resultar na desmotivação e baixa autoestima, e contribuir para repercussões nocivas à saúde dos trabalhadores ${ }^{12}$.

No que tange à distribuição das dimensões da JSS conforme o nível de escolaridade do cargo ocupado, os membros da equipe de enfermagem que ocupam cargo de nível superior apresentaram maior demanda psicológica em relação aos de cargo de nível médio $(p=0,01)$. Quanto às dimensões controle e apoio social, não houve diferença estatisticamente significativa entre os níveis dos cargos ocupados.

A percepção de maior demanda psicológica por profissionais de cargo de nível superior foi observada também em estudo realizado com profissionais de enfermagem de um hospital universitário da região centro-oeste do $\mathrm{Brasil}^{27}$ e em dois hospitais públicos do norte do Brasil ${ }^{12}$.

Uma possível explicação para essa ocorrência é o modo de organização do serviço de enfermagem no Brasil. O enfermeiro é responsável pela coordenação da equipe, gerenciamento do setor de lotação, prescrição, avaliação e execução das atividades de alta complexidade; e os técnicos de enfermagem têm suas ações voltadas para a execução das atividades prescritas e coordenadas pelo enfermeiro ${ }^{12}$. Ao realizar tais atividades, o profissional de nível superior absorve estressores relacionados ao dimensionamento inadequado de profissionais, falta de materiais e sobrecarga de trabalho, dentre outros, gerando maior demanda psicológica em relação aos técnicos de enfermagem.

Alguns autores acreditam que a percepção de alta demanda psicológica pelos enfermeiros é inerente à profissão, pois a enfermagem "lida 24 horas por dia com seres humanos doentes e que necessitam de cuidados para manutenção da vida e satisfação de suas necessidades humanas básicas"27.

Por meio da dicotomização das dimensões da JSS, os profissionais foram alocados em quatro quadrantes de acordo com o preconizado pelo modelo Demanda-Controle: trabalho de alta exigência (combinação de alta demanda e baixo controle); trabalho ativo (combinação de alta demanda e alto controle); trabalho passivo (combinação de baixa demanda e baixo controle) e trabalho com baixa exigência (combinação de baixa demanda e alto controle), conforme apresentado na Tabela 2.

TABELA 2: Distribuição dos profissionais nos quadrantes do Modelo DemandaControle $(n=123)$. Uberaba, Minas Gerais, Brasil, 2018

\begin{tabular}{lcc}
\hline Situação & $\mathbf{n}$ & $\mathbf{\%}$ \\
\hline Alta exigência & 38 & 30,6 \\
Trabalho ativo & 27 & 21,8 \\
Trabalho passivo & 24 & 19,4 \\
Baixa exigência & 35 & 28,2 \\
\hline \multicolumn{4}{l}{ Fonte: Dados da pesquisa, 2018}
\end{tabular}

A situação mais prevalente no estudo em tela foi a de alta exigência (30,6\%). Trata-se da situação mais deletéria ao organismo, estando relacionada ao surgimento de doenças físicas e mentais. A persistência nessa situação laboral pode desencadear diversos efeitos na saúde do profissional: doença cardiovascular e hipertensão; na saúde mental, especialmente depressão e ansiedade; e no sistema imunológico ${ }^{4}$.

Não há uma unanimidade em relação aos resultados encontrados na comunidade científica quanto à alocação dos profissionais nos quadrantes do modelo Demanda-Controle. As prevalências oscilam entre 20,8\% e 30,8\% para o grupo de alta exigência no trabalho; entre 18,9\% e 27,9\% para o grupo de baixa exigência; 7,6\% e 32,1\% para o quadrante de trabalho ativo; e entre19,2\% e 44,3\% para o grupo de trabalho passivo ${ }^{12,22,26,28}$. Apesar das variações encontradas, os resultados obtidos neste estudo estão semelhantes aos disponíveis na literatura.

Conforme demonstrado na Tabela 3, observou-se significância estatística na alocação dos profissionais nos quadrantes do Modelo Demanda-Controle de acordo com o setor de atuação $(p=0,02)$. Os profissionais das enfermarias e bloco cirúrgico apresentaram maiores taxas de indivíduos em trabalho de alta exigência, atingindo 41,9\% e 33,3\%, 
respectivamente; no pronto socorro, o maior índice foi de profissionais que executam trabalho ativo (50,0\%); na central de material esterilizado, os quadrantes trabalho passivo e baixa exigência apresentaram mesma prevalência (37,5\%); e no CTI o maior índice foi de trabalho de baixa exigência $(44,4 \%)$.

TABELA 3: Distribuição dos profissionais nos quadrantes do Modelo Demanda-Controle de acordo com o setor de atuação $(n=123)$. Uberaba, Minas Gerais, Brasil, 2018

\begin{tabular}{|c|c|c|c|c|c|c|c|c|c|}
\hline \multirow{3}{*}{ Setor trabalhado } & \multicolumn{8}{|c|}{ Quadrante do Modelo Demanda-Controle } & \multirow{3}{*}{$\mathbf{p}$} \\
\hline & \multicolumn{2}{|c|}{$\begin{array}{c}\text { Alta } \\
\text { Exigência }\end{array}$} & \multicolumn{2}{|c|}{$\begin{array}{c}\text { Trabalho } \\
\text { Ativo }\end{array}$} & \multicolumn{2}{|c|}{$\begin{array}{l}\text { Trabalho } \\
\text { Passivo }\end{array}$} & \multicolumn{2}{|c|}{$\begin{array}{c}\text { Baixa } \\
\text { Exigência }\end{array}$} & \\
\hline & $\mathbf{n}$ & $\%$ & $\mathbf{n}$ & $\%$ & $\mathbf{n}$ & $\%$ & $\mathbf{n}$ & $\%$ & \\
\hline Enfermarias & 18 & 41,9 & 8 & 18,6 & 8 & 18,6 & 9 & 20,9 & \\
\hline CTI & 9 & 25,0 & 4 & 11,1 & 7 & 19,4 & 16 & 44,4 & \\
\hline Bloco Cirúrgico & 6 & 33,3 & 5 & 27,8 & 5 & 27,8 & 2 & 11,1 & 0,02 \\
\hline Pronto Socorro & 4 & 22,2 & 9 & 50,0 & 0 & 0,0 & 5 & 27,8 & \\
\hline Central de Material Esterilizado & 1 & 12,5 & 1 & 12,5 & 3 & 37,5 & 3 & 37,5 & \\
\hline
\end{tabular}

Fonte: Dados da pesquisa, 2018

p: Nível de significância $(p<0,05)$

Dados de um estudo realizado no norte do Brasil corroboram com tais achados, em que a maioria dos profissionais do bloco cirúrgico está alocada no grupo de alta exigência; e a maioria dos profissionais do CTI e da central de materiais esterilizados percebe baixa exigência no trabalho ${ }^{12}$.

A situação de alta exigência no bloco cirúrgico pode ser explicada pelo fato de, na instituição em questão, serem recorrentes os casos de urgência/ emergência e os profissionais terem de executar suas ações de forma rápida e precisa. Somam-se a isto, o inadequado dimensionamento de pessoal e sobrecarga de trabalho. A presença de maior quantitativo de profissionais do CTI e central de materiais esterilizados no grupo de baixa exigência pode ser justificado pelo fato de tais profissionais possuírem alto controle nas atividades a serem desenvolvidas e pela previsibilidade das tarefas a serem realizadas.

\section{CONCLUSÃO}

Dado o exposto, e conforme a avaliação do aspecto psicossocial do ambiente de trabalho percebeu-se maior demanda psicológica nos profissionais que ocupam cargo de nível superior em relação aos profissionais de cargo de nível médio ( $p=0,01)$; e identificou-se maiores taxas de indivíduos em situação de trabalho de alta exigência nos profissionais atuantes nas enfermarias e bloco cirúrgico, atingindo 41,9\% e 33,3\%, respectivamente $(p=0,02)$.

As evidências indicam a necessidade de desenvolvimento de ações voltadas à atenção psicossocial dos profissionais de enfermagem, principalmente os de nível superior e atuantes nos setores de enfermaria e bloco cirúrgico, a fim de prevenir o adoecimento e afastamento laboral.

Ressalta-se a necessidade de reforçar o apoio social no trabalho por parte dos colegas e chefias, haja vista que tal aspecto é considerado um protetor e pode agir de modo a minimizar os efeitos negativos advindos da exposição a agentes estressores.

Compete acrescentar que este estudo apresenta limitações que devem ser consideradas na interpretação dos resultados. A generalização dos achados é limitada, uma vez que a coleta de dados foi restrita a uma única instituição hospitalar. Outra questão diz respeito ao delineamento transversal do estudo, em que há a limitação da causalidade. Ressalta-se, portanto, a importância da realização de estudos longitudinais a fim de analisar a influência dos fatores de riscos apresentados.

\section{REFERÊNCIAS}

1. Araújo AF, Bampi LNS, Cabral CCO, Calasans LHB, Queiroz RS, Vaz TS. Occupational stress of nurses from the Mobile Emergency Care Service. Rev. Bras. Enferm. [Internet]. 2020 [cited 2020 Jan 12]; 73(1):e20180898. DOI: http://dx.doi.org/10.1590/00347167-2018-0898.

2. Dejours C. Psicodinâmica do Trabalho: contribuições da escola Dejouriana à análise da relação prazer, sofrimento e trabalho. São Paulo: Atlas; 2010. 
3. Fernandes GAB, Coelho ACO, Paschoalin HC, Sarquis LMM, Greco RM. Psychological demands, control and social support in the work of community health agentes. Cogitare Enferm. [Internet]. 2018 [cited 2020 Aug 10]; (23)4: e55918. DOI: http://dx.doi.org/10.5380/ce.v23i4.55918.

4. Araújo TM, Mattos AIS, Almeida MMG, Santos KOB. Psychosocial aspects of work and common mental disorders among health workers: contributions of combined models. Rev. Bras. Epidemiol. [Internet]. 2016 [cited 2020 Jan 14]; 19(3):645-657. DOI: https://doi.org/10.1590/1980-5497201600030014.

5. Moreira IJB, Horta JA, Duro LN, Chaves J, Jacques KM, Pimentel RB, Baumhardt V, Borges DT. Psychosocial aspects of work and psychological suffering in the family health strategy. Revista de Epidemiologia e Controle de Infecção [Internet]. 2017 [cited 2020 Aug 10]; 7(1):1-7. DOI: http://dx.doi.org/10.17058/reci.v7i1.6927.

6. Greco PBT, Magnago TSB, Prochnow A, Beck CLC, Tavares JP. Use of Karasek's demand-control model in Latin America: a bibliographic research. Rev. Enferm. UFSM [Internet]. 2011 [cited 2020 Jan 25]; 1(2):272-81. DOI: https://doi.org/10.5902/217976922566.

7. Karasek RA. Job demands, job decision latitude, and mental strain: implications for job redesigning. Adm Sci Q. [Internet]. 1979 [cited 2020 Jan 10]; 24(3):285-308. DOI: http://dx.doi.org/10.2307/2392498.

8. Novaes Neto EM, Xavier ASG, Araújo TM. Factors associated with occupational stress among nursing professionals in health services of medium complexity. Rev. Bras. Enferm. [Internet]. 2020 [cited 2020 Aug 11]; 73(1):e20180913. DOI: http://dx.doi.org/10.1590/0034-7167-2018-0913.

9. Ribeiro RP, Marziale MHP, Martins JT, Galdino MJQ, Ribeiro PHV. Occupational stress among health workers of a university hospital. Rev. Gaúcha Enferm. [Internet]. 2018 [cited 2020 mar 07]; 39:e65127. DOI: https://doi.org/10.1590/19831447.2018.65127.

10. Azizah A, Rozainee K, Nada I, Izreen S, Norhafizah Z. The prevalence of occupational stress and its association with sociodemographic factors among lecturers in a private university in Malaysia. International Journal of Public Health and Clinical Sciences. [Internet]. 2016 [cited 2020 Mar 07]; 3(4):63-71. Available from: http://publichealthmy.org/ejournal/ojs2/index.php/ijphcs/article/view/326/270.

11. Sousa VFS, Araújo TCCF. Occupational Stress and Resilience among health professionals. Psicol. ciênc. prof. [Internet]. 2015 [cited 2020 Mar 12]; 35(3):900-915. DOI: https://doi.org/10.1590/1982-370300452014.

12. Petersen RS, Marziale MHP. Analysis of work capacity and stress among nursing professionals with musculoskeletal disorders Rev. Gaúcha Enferm. [Internet]. 2017 [cited 2020 Mar 12]; 38(3):1-9. DOI: https://doi.org/10.1590/1983-1447.2017.03.67184.

13. Pimenta AM, Assunção AA. Job strain and arterial hypertension in nursing professionals from the municipal healthcare network in Belo Horizonte, Minas Gerais, Brazil. Rev. Bras. Saúde Ocup. [Internet]. 2016 [cited 2020 Aug 12]; 41:1-11. DOI: http://dx.doi.org/10.1590/2317-6369000113515.

14. Gomes TDS, Puente-Palacios KE. Occupational stress, a collective phenomenon: evidence in work teams. Revista Psicologia: Organizações e Trabalho. [Internet]. 2018 [cited 2020 Aug 10]; 18(4):485-93. Available from: http://pepsic.bvsalud.org/pdf/rpot/v18n4/v18n4a05.pdf.

15. Prado CEP. Occupational stress: causes and consequences. Rev. Bras. Med. Trab. [Internet]. 2016 [cited 2020 Mar 12]; 14(3):285-9. Available from: http://www.rbmt.org.br/details/122/pt-BR/estresse-ocupacional--causas-e-consequencias.

16. Alves MGM, Chor D, Faerstein E, Lopes CS, Werneck GL. Short version of the "job stress scale": a Portuguese-language adaptation. Rev. saúde pública. [Internet]. 2004 [cited 2020 Jan 10]; 38(2):164-71. DOI: https://doi.org/10.1590/S003489102004000200003.

17. Almeida H, Brito-Costa S, Alberty A, Gomes A, Lima P, Castro FV. Occupational stress models: systematisation, analysis and description. Revista Infad de Psicología. [Internet]. 2016 [cited 2020 Aug 12]; 2(1):435-54. DOI: https://doi.org/10.17060/ijodaep.2016.n1.v2.309.

18. Karasek RA, Theörell T. Healthy work-stress, productivity, and the reconstruction of working life. New York: Basic Books; 1990.

19. Mattos AIS, Araújo TM, Almeida MMG Interaction between demand-control and social support in the occurrence of common mental disorders. Rev. Saúde Pública. [Internet]. 2017 [cited 2020 Aug 12]; 51:48. DOI: http://dx.doi.org/10.1590/s15188787.2017051006446.

20. Teixeira CAB, Gherardi-Donato ECS, Pereira SS, Cardoso L, Reisdorfer E. Occupational stress and coping strategies among nursing professionals in hospital environment. Enfermería global. [Internet]. 2016 [cited 2020 Aug 14]; 44:299-309. Available from: http://scielo.isciii.es/pdf/eg/v15n44/en_administracion3.pdf.

21. Kestenberg CCF, Felipe ICV, Rossone FO, Delphim LM, Teotonio MC. The stress of nursing workers: study in different units of a university hospital. Rev. enferm. UERJ. [Internet]. 2015 [cited 2020 Jan 10]; 23(1):45-51. DOI: http://dx.doi.org/10.12957/reuerj.2015.11487.

22. Azevedo BDS, Nery AA, Cardoso JP. Occupational stress and dissatisfaction with quality of work life in nursing. Texto \& contexto enferm. [Internet]. 2017 [cited 2020 Aug 14]; 26(1):1-11. DOI: http://dx.doi.org/10.1590/0104-07072017003940015.

23. Rodrigues CCFM, Salvador PTCO, Assis YMS, Gomes ATL, Bezerril MS, Santos VEP. Stress among nursing team members. Rev. enferm. UFPE on line. [Internet]. 2017 [cited 2020 Aug 08]; 11(2):601-8. DOI: 10.5205/reuol.10263-91568-1-RV.1102201715.

24. Trettene AS, Andrade CS, Kostrisch LMV, Tabaquim MLM, Razera APR. Stress in nursing professionals working at a specialized hospital. Rev. enferm. UFPE on line. [Internet]. 2016 [cited 2020 Aug 12]; 10 (12): 4450-8. Available from: https://periodicos.ufpe.br/revistas/revistaenfermagem/\%20article/view/11979.

25. Machado MH, Aguiar Filho W, Lacerda WF, Oliveira E, Lemos W, Wermelinger M, Vieira M, Santos MR, Souza Júnior PB, Barbosa C. Características gerais da enfermagem: o perfil sócio demográfico. Enferm. foco [Internet]. 2016 [cited 2020 Aug 08]; 7(esp) 9- 
14. Available from: http://biblioteca.cofen.gov.br/wp-content/uploads/2016/07/Caracter\%C3\%ADsticas-gerais-daenfermagem-o-perfil-s\%C3\%B3cio-demogr\%C3\%A1fico.pdf.

26. Silva JLL, Teixeira LR, Soares RS, Costa FS, Aranha JS, Teixeira ER. Estrés y factores psicosociales en el trabajo de enfermeros intensivistas. Enferm. glob. [Internet]. 2017 [cited 2020 Aug 08]; 48:80-93. DOI: http://dx.doi.org/10.6018/eglobal.16.4.249321.

27. Silva AM, Guimarães LAM. Occupational Stress and Quality of Life in Nursing. Paidéia. [Internet]. 2016 [cited 2020 Mar 12]; 26(63):63-70. DOI: https://doi.org/10.1590/1982-43272663201608.

28. Scholze AR, Martins JT, Robazzi MLCC, Haddad MCFL, Galdino MJQ, Ribeiro RP. Occupational stress and associated factors among nurses at public hospitals. Cogitare enferm. [Internet] 2017 [cited 2020 Jan 12]; 22(3):1-10. DOI: http://dx.doi.org/10.5380/ce.v22i3.50238. 